\title{
What is the association of heme aggregates with the peritrophic matrix of adult female mosquitoes?
}

Tereza Magalhaes

\begin{abstract}
The aim of this Letter is to call attention to the presence of iron-containing or heme aggregates seen within or near the peritrophic matrix (PM) in published Anopheles gambiae and Aedes aegypti midgut micrographs. The micrographs shown here suggest that the PM of An. gambiae adult female is not a homogenous layer and instead may have a peritrophin layer surrounded by heme aggregates that are possibly associated to other molecules involved in their formation. In depth studies addressing PM structure in different mosquito species are needed, as these will be important to continue clarifying the roles of the PM.
\end{abstract}

Keywords: Peritrophic matrix micrographs, Iron aggregates, Detoxifying layer, Mosquitoes

When adult female mosquitoes take a blood meal for egg production, an extracellular layer produced by midgut epithelial cells is formed around ingested blood, where it remains in the same location until it is degraded. This layer is called type 1 peritrophic matrix (PM) and its production is stimulated by distension of the midgut that naturally occurs after a blood meal [1]. The PM is composed of chitin fibrils, proteins and proteoglycans [1], which interact in an apparently sophisticated but unclear manner to form a 3-dimensional structure. The PM of Aedes aegypti and Anopheles gambiae mosquitoes differs in a few aspects such as formation kinetics, and the number and mode of production of proteins. While the An. gambiae PM appears to have approximately 40 major proteins, fewer PM proteins have been identified in Ae. aegypti (around 20) [2]. An in depth proteomic analysis of the An. gambiae PM broadened the number of PM proteins in this species [3], and it is possible the same could occur with Ae. aegypti. As for protein production, An. gambiae PM proteins are stored in vesicles of epithelial cells and are promptly secreted after a blood meal, while in Ae. aegypti PM proteins need to be synthesized after blood ingestion [2]. Following an increase in the number of studies on insect PMs, different types of molecular structure models have

\footnotetext{
Correspondence: teca@cpqam.fiocruz.br

Department of Virology and Experimental Therapeutics, Centro de Pesquisas Aggeu Magalhães, Fundação Oswaldo Cruz, Av. Moraes Rego s/n - Cidade
} Universitária, CEP 50740-465 Recife, PE, Brazil been proposed $[3,4]$. These models are based on the characterization of PM proteins and their putative interaction with molecules such as chitin fibrils and digestive enzymes secreted by the midgut epithelium. They also consider the putative functions of insect PMs, such as controlling the traffic of molecules between midgut epithelium and lumen, and protection of the midgut epithelium against mechanical abrasion and toxic products derived from blood digestion (e.g. heme). Whilst several putative roles have been ascribed to the PM of different mosquito species, various aspects of the molecular structure and functions of mosquito PM remain undefined. For instance, a study using Ae. aegypti shows no effect on mosquito survival, fecundity or protection against pathogens when PM formation is hindered [5]. Results on the protective role of mosquito PM against pathogens have also varied among studies.

The mosquito PM is a physical structure and can be physically isolated. In regards to PM micrographs, several studies show the mosquito midgut epithelium and PM under different conditions/treatments and magnification. As there is no described standard staining for insect PMs, authors have used different staining methodologies and visualized it as a sheet between the midgut epithelium and blood bolus. The appearance and description of the PM varies among studies, even within the same mosquito species, as different markers interact with different PM molecules. Pascoa et al. [6] demonstrated the possible role of Ae. aegypti PM to serve as a 
detoxifying barrier. In this study, the authors state that in an unstained micrograph $(240 \times$ magnification) the PM looks darker than the midgut epithelium and ingested blood because of heme bound to it. Heme is derived from hemoglobin digestion. When Ae. aegypti midgut is stained with 3,3'-diaminobenzidine (DAB) for detection of heme peroxidase activity, the reaction produces a dark brown precipitate seen by light microscopy, which confirms the authors' assumption that heme is bound to the PM. In this study, the PM is referred to whenever heme peroxidase activity is observed. Micrographs (640× magnification) of Ae. aegypti PM at several time-points (11, 22, 30, and $48 \mathrm{~h}$ ) post blood meal (pbm) show a darker sheet between the midgut epithelium and ingested blood in the lumen. At $11 \mathrm{~h}$ pbm the PM looks thin and two dark layers can be seen. At $48 \mathrm{~h}$, corresponding to a timepoint where blood digestion is in an advanced stage, the dark staining looks amorphous throughout the midgut lumen, as if the PM is already degraded. Another study shows the association of an Ae. aegypti mucin-like molecule (AeIMUC1) to the PM. In electron micrographs of midguts dissected at 12 and $24 \mathrm{~h} \mathrm{pbm}$ the PM is seen between the midgut epithelium and midgut lumen with heme aggregates observed "below" it [7]. Thus, according to the authors, these aggregates are not associated to the PM, which may sound contradictory to the PM-associated heme peroxidase activity in Ae. aegypti demonstrated by Pascoa et al. [6].

As for An. gambiae PM, Devenport et al. [8] shows the PM as a thin and undefined layer in midguts dissected at 12 and $24 \mathrm{~h}$ pbm (electron micrographs). In these micrographs, authors call attention to dark aggregates seen in the lumen near the PM and attribute these aggregates to "iron-containing byproducts of digestion". In this case, the authors also do not associate the aggregates to the PM. The same description is found for aggregates observed near An. gambiae PM in Devenport et al. [9], however, one could claim that the aggregates can be seen within the PM in some of the micrographs.

Here, two types of An. gambiae micrographs are shown. In the first (Figure 1, 200× magnification), tissues were embedded in paraffin and incubated with mouse polyclonal anti-AgAper1 sera and then with anti-mouse IgG antibody conjugated with FITC as secondary antibody. In these samples, a thin green fluorescent layer corresponding to An. gambiae peritrophin 1 staining can be observed, similar to the micrographs published by Devenport et al. [8] showing stained AgAper1 within the mosquito PM.

The second type of micrograph (Figure 2) shows An. gambiae midguts dissected at $24 \mathrm{~h}$ and $48 \mathrm{~h}$ postblood meal, embedded in Araldite and stained with toluidine blue $(1000 \times$ magnification). In these, the layer

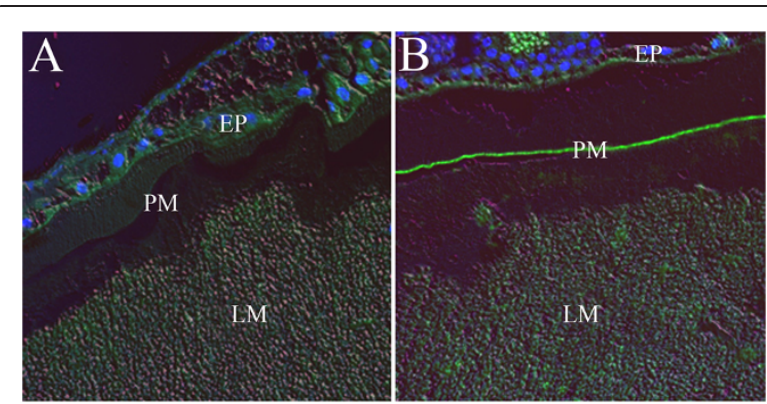

Figure 1 Immunofluorescence of Anopheles gambiae abdomen at $24 \mathrm{~h}$ post-blood meal. Paraffin-embedded sections were incubated with sera from naive mice $(\mathbf{A})$ or mice immunized with AgAper1 (B) as primary antibody, and with anti-mouse IgG-FITC as secondary antibody. Arrow in B shows AgAper1 fluorescence within the peritrophic matrix. Mounting media containing DAPI was used for nuclear staining of epithelial cells. Images were taken with a monochromatic camera using DIC, $480 \mathrm{~nm}$, and $546 \mathrm{~nm}$ light. They were then false-coloured and merged to produce a single image. EP: epithelium; PM: peritrophic matrix; LM: midgut lumen containing undigested red blood cells. Magnification: 200x.

between the midgut epithelium and midgut lumen, which probably corresponds to the PM, looks much thicker than the AgAper1 immunofluorescence and contains what appear to be heme aggregates.

Taken together, the micrographs shown here suggest that: the localization and distribution of An. gambiae

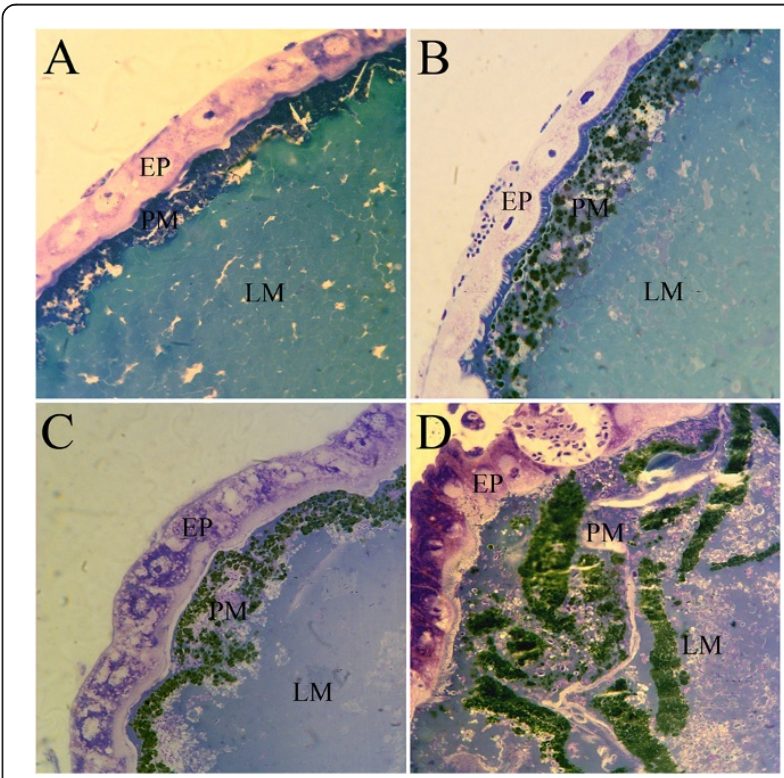

Figure 2 Light micrographs of Anopheles gambiae midguts dissected at $24 \mathrm{~h}$ ( $A$ and $C$ ) or at $48 \mathrm{~h}$ post-blood meal (B and D). Sections were immersed in Araldite and stained with toluidine blue after sectioning. EP: epithelium; PM: peritrophic matrix; LM: midgut lumen. Magnification: 1000x. 
peritrophin 1 , and possibly of other peritrophins, does not reflect the full extent of the PM, which is reasonable to assume; and that the PM is not an homogenous layer, but may have a peritrophin layer surrounded by heme aggregates that are possibly associated to other molecules involved in the formation of these aggregates.

This letter does not intend to prove that heme is associated to An. gambiae PM, but to raise questions in regards to this issue considering the presence of ironcontaining (as some authors name it) or heme aggregates seen in various An. gambiae and Ae. aegypti midgut micrographs. In depth studies addressing PM structure in both mosquito species are needed, including experiments on chitin versus peritrophin staining, as these will be important to continue clarifying the roles of the PM in these insects.

\section{Competing interests}

The author declares that she has no competing interests.

\section{Authors' contribution}

TM performed the experiments and wrote the manuscript.

\section{Acknowledgements}

Experiments shown here were performed at the Human Health Division at the International Centre of Insect Physiology and Ecology (ICIPE) based in Nairobi, Kenya, and at the Arthropod-borne and Infectious Diseases Laboratory (AIDL) at Colorado State University (CSU), Fort Collins-CO, USA. The author would like to thank the colleaques from these institutions, in special Dr. John Githure, Chimtawi, Milka and Jeremiah from ICIPE, and Dr. Brian D. Foy from AIDL. A special thanks also to Dr. John C. Beier (former advisor) from the Department of Public Health Sciences at the University of Miami, Miami-FL, USA, and Dr. Wilfred E. Injera (former PhD colleague).

Received: 16 January 2014 Accepted: 7 August 2014

Published: 13 August 2014

\section{References}

1. Tellam RL: The peritrophic matrix. In Biology of the Insect Midgut. Edited by Lehane MJ, Billingsley PF. London: Chapman \& Hall; 1996.

2. Devenport M, Jacobs-Lorena $M$ : The peritrophic matrix of hematophagous insects. In The Biology of Disease Vectors. 2nd edition. Edited by Marquardt WC. Burlington MA: Elsevier Academic Press; 2005.

3. Dinglasan RR, Devenport M, Florens L, Johnson JR, McHugh CA, DonnellyDoman M, Carucci DJ, Yates JR III, Jacobs-Lorena M: The Anopheles gambiae adult midgut peritrophic matrix proteome. Insect Biochem Mol Biol 2009, 39(2):125-134

4. Hegedus D, Erlandson M, Gillott C, Toprak U: New insights into peritrophic matrix synthesis, architecture, and function. Annu Rev Entomol 2009, 54:285-302.

5. Kato N, Mueller CR, Fuchs JF, McElroy K, Wessely V, Higgs S, Christensen BM: Evaluation of the function of a type I peritrophic matrix as a physical barrier for midgut epithelium invasion by mosquito-borne pathogens in Aedes aegypti. Vector Borne Zoonotic Dis 2008, 8(5):701-712.

6. Pascoa V, Oliveira PL, Dansa-Petretski M, Silva JR, Alvarenga PH, JacobsLorena M, Lemos FJ: Aedes aegypti peritrophic matrix and its interaction with heme during blood digestion. Insect Biochem Mol Biol 2002, 32(5):517-523.

7. Devenport M, Alvarenga PH, Shao L, Fujioka H, Bianconi ML, Oliveira PL, Jacobs-Lorena M: Identification of the Aedes aegypti peritrophic matrix protein AeIMUCl as a heme-binding protein. Biochemistry 2006, 45(31):9540-9549.
8. Devenport M, Fujioka H, Jacobs-Lorena M: Storage and secretion of the peritrophic matrix protein Ag-Aper1 and trypsin in the midgut of Anopheles gambiae. Insect Mol Biol 2004, 13(4):349-358.

9. Devenport M, Fujioka H, Donnelly-Doman M, Shen Z, Jacobs-Lorena M Storage and secretion of Ag-Aper14, a novel peritrophic matrix protein, and Ag-Muc1 from the mosquito Anopheles gambiae. Cell Tissue Res 2005, 320(1):175-185.

doi:10.1186/1756-3305-7-362

Cite this article as: Magalhaes: What is the association of heme aggregates with the peritrophic matrix of adult female mosquitoes? Parasites \& Vectors 2014 7:362.

\section{Submit your next manuscript to BioMed Central and take full advantage of:}

- Convenient online submission

- Thorough peer review

- No space constraints or color figure charges

- Immediate publication on acceptance

- Inclusion in PubMed, CAS, Scopus and Google Scholar

- Research which is freely available for redistribution 